\section{Multiple abdominal abscesses complicated by severe sepsis as a result of occult Crohn's disease}

\author{
Antonio Vannini, ${ }^{1}$ Luca Croci, ${ }^{2}$ \\ Elena Guidetti, ${ }^{2}$ Francesco Tovoli, ${ }^{2}$ \\ Fabio Cassani, ${ }^{3}$ Luigi Bolondi ${ }^{2}$ \\ 'Department of Emergency Medicine, \\ S. Orsola-Malpighi Hospital, Bologna; \\ 2Department of Medical and Surgical \\ Sciences, University of Bologna; \\ ${ }^{3}$ Department of Digestive Diseases and \\ Internal Medicine, S. Orsola-Malpighi \\ Hospital, Bologna, Italy
}

\begin{abstract}
Intra-abdominal infections represent an important cause of mortality in worldwide population and often require both rapid diagnostic work-up and swift therapeutic decisions. In this paper a relatively frequent pathologic condition in industrialized countries is described as a potential cause of multiple abdominal abscesses with severe sepsis. In the subsequent review of the literature, first-line diagnostic examinations and therapeutic options, both medical and surgical, are discussed according to the most recent guidelines and recommendations.
\end{abstract}

\section{Introduction}

Intra-abdominal sepsis is a complex, multifactorial, evolutive syndrome that can progress to conditions of varying severity. If improperly treated, it may cause the functional impairment of one or more vital organs or systems, which could lead to multiple organ failure. ${ }^{1}$ Despite advances in diagnosis, surgery and antimicrobial therapy, mortality rates associated with complicated intra-abdominal infections remain extremely high. ${ }^{2}$

We report a case of a previously healthy young male presenting with multiple liver abscesses (LAs) and a right psoas muscle abscess (PMA) who developed a condition of severe sepsis with an incipient multi-organ failure. After clinical stabilization of the patient by means of intensive fluid resuscitation, wide spectrum antibiotics and percutaneous aspiration of the abscesses, instrumental examinations led to a partially unexpected diagnosis of complicated Crohn's disease (CD).

\section{Case Report}

A 27-year-old male was admitted to our hospital complaining fatigue, nausea, dyspnea, palpitations and sweating episodes in the last few days. He reported a progressive weight loss in recent years, however he did not complain diarrhea or abdominal pain. His past medical history was not significant, expect for a surgical intervention for an anal fistula and a moderate consumption of alcohol (approximately $40 \mathrm{~g} /$ day).

On physical examination he had hypotension, tachycardia and pale skin. He was apyretic and eupnoic on room air (blood pressure 90/65 mmHg, heart rate $115 \mathrm{bpm}$, oxygen saturation $96 \%$ while breathing on room air). Laboratory tests were significant for elevated markers of flogosis and severe microcitic anemia (white blood cell $18,000 / \mathrm{mmc}$ with a left shift, C-reactive protein $24 \mathrm{x}$ upper normal limit, hemoglobin $6.5 \mathrm{~g} / \mathrm{dL}$, mean corpuscular volume $71 \mathrm{fL}$, creatinine $0.9 \mathrm{mg} / \mathrm{dL}$ ). Blood, urine and stool coltures were negative. Parasitological examinations of stools were also negative. Serological study for hepatitis B and $\mathrm{C}$ virus, and HIV were negative. Neoplastic markers, including CA 19-9, CEA and alphafetoprotein, were normal. Abdominal ultrasound (US) examination revealed liver enlargement with multiple hypoechoic nodular lesions in right lobe (Figure 1), a small amount of intraperitoneal fluid and right pleural effusion. In addition, wall thickening of the terminal ileum and the transverse colon was also noted. A contrast-enhanced computerized tomography (CT) of the abdomen confirmed several hypodense lesions throughout the liver highly suggestive for LAs and a further small hypodense lesion within right ileo-psoas muscle.

The patient was treated with a combined intravenous antibiotic therapy including third generation cephalosporins and fluoroquinolones. However, his clinical conditions failed to improve and three days after admission he suffered from acute respiratory failure and oliguria (hemogasanalysis on room air: $\mathrm{pH}$ $7.35, \mathrm{pO}_{2} 45 \mathrm{mmHg}, \mathrm{pCO}_{2} 42 \mathrm{mmHg}$, bicarbonate $22 \mathrm{mmol} / \mathrm{L}$ ). Treated with intensive fluid resuscitation, repeated blood transfusions and potentiated antibiotic therapy (piperacillin/tazobactam, clindamycin and ciprofloxacin), in the following days the patient underwent multiple percutaneous drainages of the major LAs. Cultures of the fluid were positive for a strain of Enterococcus faecalis sensitive to the ongoing antibiotic therapy. Neither amebae nor acid fast bacilli were found. A trans-thoracic echocardiogram was not significant for endocarditic vegetations. A positron emission tomography showed moderately increased uptake in the right lobe
Correspondence: Francesco Tovoli, Department of Medical and Surgical Sciences, University of Bologna, via Massarenti 9, 40138 Bologna, Italy. Tel. +39.051.6362713 - Fax: +39.051.6362240.

E-mail: francesco.tovoli2@.unibo.it

Key words: intra-abdominal infections, sepsis, Crohn disease, liver abscess, psoas abscess.

Contributions: AV, revised the final draft; LG and EG, revised the literature about the topic; FT, wrote the draft; $\mathrm{FC}$, responsible for patient's care and contributed to draft writing; LB, revised the final draft.

Conflict of interests: the authors declare no potential conflict of interests.

Received for publication: 4 March 2014.

Revision received: 26 April 2014.

Accepted for publication: 15 May 2014.

This work is licensed under a Creative Commons Attribution 3.0 License (by-nc 3.0).

(C) Copyright A. Vannini et al., 2014

Licensee PAGEPress, Italy

Emergency Care Journal 2014; 10:3221

doi:10.4081/ecj.2014.3221

of the liver and in the right ileo-psoas muscle. An abdominal CT focused on small and gross bowel, carried out with both intravenous and intraluminal contrast, showed terminal ileum and right colon wall thickening with bowel wall enhancement, highly suggestive for CD.

A week after complete stabilization we performed a confirmative endoscopic examination. Colonscopy showed hyperemic, oedematous and fragile mucosa of terminal ileum and caecum, with serpiginous ulcers and pseudopolyps, alternate with stretches of normal mucosa. Histological examination of the biopses was highly suggestive for Crohn disease. The patient was discharged in good health 43 days after his admission. He was referred to a tertiary referral center and proposed for surgery, but he was immediately lost to follow-up.

\section{Discussion}

This case underlines the role of pyogenic LAs as a cause of sepsis, even in the younger population. Differential diagnosis of LAs are reported in Table 1.

LAs are a rare complication of $\mathrm{CD}$, their prevalence ranging from 114 to 297 per 100,000 CD patients (10-15 times less than what found in the general population). ${ }^{3}$ LAs are usually encountered in younger CD patients with a longstanding disease (mean 
age 36.5 years at the diagnosis). ${ }^{4}$ Underlying mechanisms favoring LAs development in CD patients include portal bacteriemia (secondary to disruption of mucosal barrier, chronic corticosteroid treatment and possibly malnutricion), indirect extension by biliary involvement in the setting of coexisting biliary diseases (including primary sclerosing cholangitis and choledocholithiasis), septic embolization by the combination of portal-mesenteric thrombosis and infection, facilitated by chronic steroid treatment and longstanding inflammation. ${ }^{3}$ Despite portal pyemia is frequently found in CD as a consequence of the chronic alteration of mucosal integrity, the development of pyogenic LAs remains a rare event.

PMAs are also considered as a possible complication of CD but, similarly to LAs, they seldom represent the first finding leading to a diagnosis of an inflammatory bowel disorder. ${ }^{5}$ They can be usually found in patients with a previous or ongoing history of steroid drugs consumption, occurring in a range from 10 months to 20 years after CD diagnosis. ${ }^{6}$ On the other hand, nowadays, $\mathrm{CD}$ represents the most frequent cause of PMA in immunocompetent patients. $^{7}$

Both LAs and PMA are rare and challenging conditions, since they share a significant mortality and their usual clinical presentation is subtle and aspecific (fever, abdominal pain). LAs can also be symptomatic for hepatomegaly, jaundice and ascites; similarly, hip pain, difficulty in walking and pain in the buttock or thigh should arouse suspicion of PMA. However, clinical features are variable, depending on the size of the abscess, general health of the patient, associated diseases and complications. C-reactive protein and erythrocyte sedimentation rate are almost invariably elevated and also leucocytosis with left shift is usually present. ${ }^{5}$ Abdominal US represent a first-line examination which can potentially detect both LAS and PMA; however, CT remains the main diagnostic technique for PMA as it can provide important information about abscess extension, concurrent gastrointestinal involvement and possible infiltration of perivisceral adipose tissue. ${ }^{7,8}$ Furthermore, contrast enhanced CT may offer useful information in differentiating LAs from other liver focal lesions.

Therapeutic management of CD-related abscesses include both antibiotic therapy and transcutaneous or surgical management.

Initial antibiotic therapy is typically empirical because the patient needs immediate attention and microbiological data (culture and susceptibility results) can require up to 48 $\mathrm{h}$ before they are available for a more detailed analysis. ${ }^{2}$ Depending on the range requirements of antimicrobial coverage, multiple antimicrobial regimens are available. Beta-lactam/beta-lactamase inhibitor combinations exhibit in vitro activity against gram-positive, gram-negative, and anaerobic organisms ${ }^{9,10}$ and are viable options for empirical treatment of intra-abdominal infections. ${ }^{9}$ Furthermore, given their excellent tissue penetration and strong activity against aerobic gram-negative bacteria, fluoroquinolones have been widely used in recent years for treatment of intraabdominal infections. ${ }^{2}$ Tigecycline and car- bapenems offer a wide spectrum of antimicrobial activity, however it should be noted that increased carbapenem consumption has been associated with an increased emergence of carbapenem resistance among Enterobacteriacea, particularly in Klebsiella pneumoniae. For this reason most recent guidelines for management of intra-abdominal infections suggest to limit carbapenems use to

Table 1. Differential diagnoses of pyogenic liver abscesses classified according to their pathogenic mechanisms.

\begin{tabular}{lc} 
Pathogenic mechanism & Pathologies \\
Biliary tract disease & Cholecystitis \\
& Primary sclerosing cholangitis \\
& Ascending cholangitis \\
Inflammatory bowel disorders & Diverticulitis \\
Portal dissemination & Gut perforation \\
& Appendicitis \\
& Amoebic infection \\
& Other parassitosis \\
Arterial dissemination & Abdominal surgery \\
Septic embolization (combination of chronic steroid & Inflammatory bowel disorders \\
treatment and longstanding inflammation) & Infective endocarditis \\
\hline Direct spread & Inflammatory bowel disorders \\
\hline
\end{tabular}

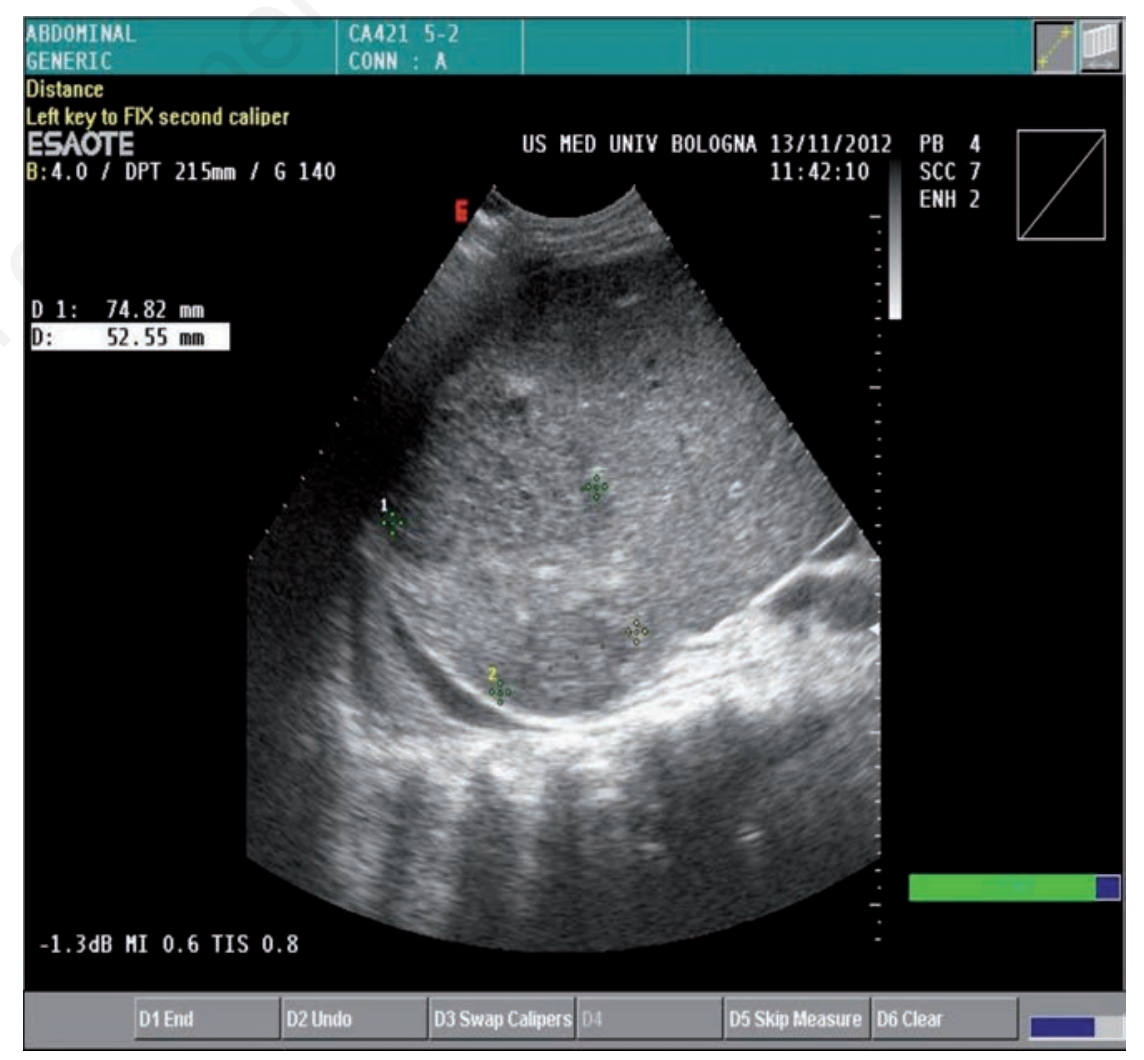

Figure 1. Large hypoechoic nodular lesions in liver right lobe detected by abdominal ultrasound. 
patients with prior exposure to antibiotics or serious comorbidities requiring concurrent antibiotic therapy or with healthcare-associated infections. ${ }^{7}$ Drainage of the abscesses, percutaneous or surgical, should also be considered. As regards large PMAs, drainage should always be performed. ${ }^{5}$ Selection between percutaneous or surgical approach is to be evaluated in every single case. As a rule of thumb, surgical drainage represents the elective treatment for patients with CD-related abscesses, while percutaneous drainage should be reserved to unstable and/or septic patients. ${ }^{11}$ However, it is widely accepted that percutaneous management is not indicated in cases of multiloculated abscesses, as a difficult approach to the abscessual cavity or presence of overt fistulae. ${ }^{11}$

Our case presents some peculiar and unusual characteristics. First of all clinical presentation of our patient is almost unique, as cooccurrence of both LAs and PMA has been described in a single case report (notably, it referred to a 24-year-old male with an already known diagnosis of CD, who had been previously treated with salazopyrin). ${ }^{12}$ The extension and the multifocality of the absesses probably conditioned the particular severity of the clinical presentation of disease in our patient, to the extent of a condition of incipient multiorgan failure. It is also to underline that our patient was never pyretic, in absence of any cause of immunocompromisation other than his septic condition itself. To our knowledge, such severe onset of disease has never been reported in patients with CD-related LAs. In our patient an early wide-spectrum antibiotic therapy, aggressive fluid infusion and percutaneous drainage of major LAs avoided a fatal outcome; however it should be noted that complete clinical stabilization took many weeks and discharge was realizable more than 40 days after admission.

\section{Conclusions}

CD is a rare but possibile cause of disseminated septic abscesses, possibly leading to severe sepsis and incipient multi-organ failure. In this setting, practical consequences deriving from a correct diagnosis in terms of survival, disabilities and disease relapses are particularly evident, as CD-related abscesses are extremely more frequent in young patients. Consequently, once multiple abscesses are detected in a septic patient, differential diagnosis should include CD and, if clinical suspect is very strong, endoscopy and specific radiological studies should be performed once clinical conditions are stabilized.

\section{References}

1. Levy MM, Fink MP, Marshall JC, et al. 2001 SCCM/ESICM/ACCP/ATS/SIS international sepsis definitions conference. Crit Care Med 2003;31:1250-6.

2. Sartelli M, Viale P, Catena F, et al. 2013 WSES guidelines for management of intraabdominal infections. World J Emerg Surg 2013;8:3.

3. Aguas M, Bastida G, Nos P, et al. Septic thrombophlebitis of the superior mesenteric vein and multiple liver abscesses in a patient with Crohn's disease at onset.
BMC Gastroenterol 2007;7:22.

4. Karaca C, Pinarbasi B, Danalioglu A, et al. Liver abscess as a rare complication of Crohn's disease: a case report. Turk J Gastroenterol 2004;15:45-8.

5. Marín I, Serra I, Mañosa M, et al. Psoas abscess as a complication of Crohn's disease: report of three cases and literature review. Gastroenterol Hepato 2009;32:55761.

6. Zapata E, Cosme A, Ojeda E, et al. Absceso de psoas complicando la enfermedad de Crohn: estudio de pacientes. Rev Esp Enferm Dig 2006;98:391-5.

7. Lobo DN, Iftikhar SY, Dunn WK, Sholefield JH. Psoas abscesses complicating colonic disease: imaging and therapy. Ann Roy Coll Surg 1998;80:405-9.

8. Zissin R, Gayer G, Werner M, et al. Iliopsoas abscess: a report of 24 patients diagnosed by CT. Abdom Imaging 2001;26:533-9.

9. Powell LL, Wilson SE. The role of beta-lactam antimicrobials as single agents in treatment of intra-abdominal infection. Surg Infect (Larchmt) 2000;1:57-63.

10. Lode HM. Rational antibiotic therapy and the position of ampicillin/sulbactam. Int $\mathrm{J}$ Antimicrob Ag 2008;32:10-28.

11. Jawhari A, Kamm MA, Ong C, et al. Intraabdominal and pelvic abscess in Crohn's disease: results of non-invasive and surgical management. Brit J Surg 1998;85:36771.

12. Shinohara T, Yamashita Y, Watanabe K, et al. A case of Crohn's disease complicated by liver abscess and iliopsoas abscess. Rinsho Geka Gakkai Zasshi 2003;64:67882. 